\title{
Looking for a better definition and diagnostic strategy for acute kidney injury: a new proposal
}

Even though there are many definitions of acute kidney injury (AKI), it is classically defined as a heterogeneous group of conditions characterized by a sudden decrease in glomerular filtration rate (GFR), expressed as an increase in serum creatinine (serum creatinine $\geq 0.3 \mathrm{mg} / \mathrm{dl}$ within 48 hours or serum creatinine $\geq 1.5$ times baseline within the prior 7 days) or urine volume reduction $(<0.5 \mathrm{ml} / \mathrm{kg} / \mathrm{h} \text { for } 6 \text { hours })^{1,2}$ However, this definition does not exactly reflect the whole pathophysiology of this syndrome, since an acute renal injury can be limited to the tubules without compromising the glomeruli, and consequently reducing neither the patient's GFR nor his/ her diuresis. This phenomenon is clinically observed in some drug-induced kidney injuries (eg: aminoglycosides) or in partial kidney ischemia, since renal medulla is more sensitive to hypoxia than renal cortex..$^{3,4}$ Therefore, the current AKI definition can avoid its diagnosis in some particular cases. In addition, there are two additional inconveniences derived from the current AKI definition: Firstly, non oliguric AKI diagnosis is based on detecting a GFR reduction but the diagnostic marker usually used is serum creatinine, which is a late marker due to the fact that it rises after a GFR reduction of at least $50 \%$. This fact inexorably leads to a late AKI diagnosis, delayed treatment and worse prognosis. ${ }^{1,3}$ Secondly, despite having described novel and earlier AKI biomarkers, they have reduced availability, low specificity, usefulness not in all causes of AKI, insufficient patient outcome and cost-effectiveness studies, and mainly they do not guarantee the immediate AKI detection since their request is subject to the clinical suspicion of this condition by the attending physician, which definitely depends on the AKI definition currently used..$^{5,6}$ In order to overcome these difficulties we present here the following proposals, valid for both the adult and pediatric patient.

Firstly, to change the current AKI diagnosis definition to a more complete one, such as: Acute kidney injury is a heterogeneous group of conditions characterized by a sudden alteration of tubular function, decrease in GFR or urine volume reduction. The idea of modifying AKI definition is not just for academic reasons but mainly pragmatic ones, since this definition would lead the physician to evaluate not only glomerular but also tubular function in order to rule out the presence of this condition, contributing in this more meticulous evaluation to a more sensitive and earlier detection of this syndrome. Secondly, early AKI diagnosis should be based not just on monitoring a serum GFR marker but also a urinary tubular functional marker, for instance fractional excretion of sodium (FENa), and also to do it before AKI installation, performing a preventive renal monitoring. Of course, this strategy is proposed to be applied to high risk AKI patients (eg: critical care patients or individuals on potentially renal damaging drugs or conditions). The idea would be not looking for a particular absolute value of urinary index (eg: Miller's classical indexes ranges) or distinguishing between pre-renal and renal AKI, but looking for a significant change (increase or decrease) in a urinary index value respect to its basal values. It could be argued that these preventively evaluated urinary indexes changes could be caused by just hemodynamic fluctuations, but an adequate screening test should be sensitive and can have false positive results. Moreover, these hemodynamic fluctuations if they are not early detected and adequately managed they can induce renal ischemic damage. Thus, the early detection of these risky clinical settings by applying the preventive renal monitoring could precisely help to avoid AKI. In this sense, we have preliminary data which can support this idea obtained from a prospective study we are currently performing. In this protocol, daily FENa was obtained in every patient admitted in the critical care unit since his/her admission, and those patients who presented a significant change in the FENa the day before (day - 1) the clinical diagnosis of AKI (day 0), they finally developed AKI. The preventive renal monitoring could early detect the appearance of AKI at a moment when an exhaustive evaluation of his/ her volume status and nephrotoxic medication could avoid or mitigate this syndrome. ${ }^{7}$ Of course, we welcome researchers from other centers who would like to join our study. In conclusion, we propose to modify acute kidney injury definition and diagnostic strategy, including the concepts of 
acute tubular dysfunction, and preventive renal monitoring, respectively, in order to make a wider and earlier diagnosis of this syndrome.

Carlos G. Musso, MD. PhD ${ }^{a, b}$, Sergio Terrasa, $M D^{c}$, Mariana Ciocchini, $\mathrm{MD}^{d}$, Henry Gonzalez-Torres $M g^{e}$ and Gustavo Aroca-Martinez MD. PhD

1. Physiology Department. InstitutoUniversitario delHospital Italiano de Buenos Aires, Argentina.

2. Ageing Biology Unit. Hospital Italiano de Buenos Aires. Argentina.

3. Family MedicineService. Hospital Italiano de Buenos Aires, Argentina.

4. Daomi Institute affiliated to Facultad de Medicina, Universidad de Buenos Aires, Buenos Aires, Argentina.

e. Clínica de la Costa, Universidad Simón Bolivar, Barranquilla, Colombia.

E-mail adress: carlos.musso@hospitalitaliano.org.ar

http:/ / dx.doi.org/10.5546/ aap.2019.eng.4

To cite: Musso CG, Terrasa S, Ciocchini M, González-Torres H, et al. Looking for a better definition and diagnostic strategy for acute kidney injury: a new proposal. Arch Argent Pediatr 2019;117(1):4-5.

\section{REFERENCES}

1. Levey AS, James MT. Acute Kidney Injury. Ann Intern Med. 2018; 168(11):837.

2. KDIGO Clinical Practice Guideline for Acute Kidney Injury. Kidney Int. 2012; 2(1):1-138.

3. Rennke H, Denker B. Renal Pathophysiology. Philadelphia: Lippincott Williams \& Wilkins; 1994.Págs.267-90.

4. Musso CG. Aminoglycosides and nephropathy. Rev Electron Biomed. 2004; 2:2-4.

5. Beker BM, Corleto MG, Fieiras C, Musso CG. Novel acute kidney injury biomarkers: their characteristics, utility and concerns. Int Urol Nephrol. 2018; 50(4):705-13.

6. Pacheco-Lugo L, Díaz-Olmos Y, Aroca-Martínez G. Biomarkers in biological fluids and their potential use as indicators of lupus nephritis in individuals with systemic lupus erythematous. Rev Colomb Nefrol. 2016; 3(1):39-47.

7. Musso CG, Silva D, Arroyo M, Propato F, et al. Renal monitoring in intensive care unit. Abstract presentado en XII Congreso Internacional de la ISHD; del 18 al 21 de abril de 2018. Cartagena de Indias, Colombia. 2018. 